Itinéraires Itinéraires

Littérature, textes, cultures

2018-1| 2018

Le manifeste à travers les arts : devenirs d'un genre indiscipliné

\title{
De la théorie à la pratique : « Le manifeste des écrivains et artistes révolutionnaires » (1932)
}

From Theory to Practice: "The Manifesto of the Revolutionary Writers and Artists" (1932)

\section{Gwenn Riou}

\section{OpenEdition}

\section{Journals}

Édition électronique

URL : http://journals.openedition.org/itineraires/4212

DOI : $10.4000 /$ itineraires. 4212

ISSN : 2427-920X

Éditeur

Pléiade

\section{Référence électronique}

Gwenn Riou, « De la théorie à la pratique : « Le manifeste des écrivains et artistes révolutionnaires » (1932) », Itinéraires [En ligne], 2018-1 | 2018, mis en ligne le 15 septembre 2018, consulté le 15 mai 2020. URL : http://journals.openedition.org/itineraires/4212 ; DOI : https://doi.org/10.4000/itineraires. 4212

Ce document a été généré automatiquement le 15 mai 2020.

\section{c) (1)}

Itinéraires est mis à disposition selon les termes de la licence Creative Commons Attribution - Pas d'Utilisation Commerciale - Pas de Modification 4.0 International. 


\title{
De la théorie à la pratique : « Le manifeste des écrivains et artistes révolutionnaires » (1932)
}

\author{
From Theory to Practice: "The Manifesto of the Revolutionary Writers and \\ Artists" (1932)
}

\section{Gwenn Riou}

Le 13 décembre 1931, l'écrivain et critique d'art communiste Léon Moussinac écrit :

[...] il est urgent que l'on fixe la situation réelle de la section française des écrivains et des artistes révolutionnaires... Nous laissons ainsi, par notre faute, aller au fascisme des éléments intellectuels que nous aurions pu amener à la lutte des classes révolutionnaires en leur faisant comprendre notre doctrine et la théorie révolutionnaire du marxisme-léninisme... La section française des écrivains et artistes révolutionnaires doit être dirigée par le Parti. Il n'y a aucune raison pour qu'il en soit autrement. Le Parti doit s'en occuper tout de suite. (Moussinac cité par Klein $1988: 17-18)$

2 Quelques mois plus tard, le 17 mars 1932, l'Association des écrivains et artistes révolutionnaires (AEAR) est créée sous la présidence d'honneur de Léopold Averbach, de Théodore Dreiser, de Maxime Gorki, d'André Marty, de Romain Rolland et d'Edmond Fritsch. Le bureau est composé de Paul Vaillant-Couturier (secrétaire général), Jean Fréville (secrétaire-adjoint) et Léon Moussinac (trésorier). Dès sa création, l'association revendique « 200 adhérents environ (dont 80 pour les écrivains, 120 pour les artistes). [La] fraction communiste comprend 36 camarades $^{1} »$ (Fréville cité par Morel 1985 : 413).

3 Le Parti communiste est donc à l'origine de la création de l'AEAR, plus précisément, c'est le Bureau international pour la littérature révolutionnaire - qui est lui-même une création de l'Internationale communiste - qui pose les bases de l'association ${ }^{2}$. De cette façon, lorsqu'est publié le manifeste de l'organisation dans L'Humanité on peut y lire :

Détruire. Organiser. Pour nous l'un ne va pas sans l'autre. C'est-à-dire que le prolétariat doit poser dès à présent les fondements d'une culture prolétarienne qui ne pourra s'épanouir qu'au lendemain de la victoire de la Révolution socialiste, 
mais qui, dès l'époque de l'impérialisme, doit servir à préparer cette victoire 3 .

(Anonyme [Paul Vaillant-Couturier] $1932: 4$ )

Deux mondes s'affrontent, "l'impérialisme pourrissant » et «le jeune socialisme ». Il s'agit donc de prendre part à cette lutte de classe et de détruire cet impérialisme dont le symbole est la décadence de la culture bourgeoise. Le prolétariat doit alors, « relever la culture mondiale ", que l'impérialisme a mise à mal et dans ce combat « c'est en liant le travail théorique ; littéraire, artistique, à la pratique révolutionnaire qu'écrivains et artistes mériteront le titre de lutteurs du prolétariat».

5 Le vocabulaire employé ici est caractéristique de la période dite de «stalinisation » du PCF et de sa ligne «classe contre classe » qui prévaut jusqu'à la fin des années 1920. Cette pratique est cependant remise en question car le Parti - qui s'apparente alors à une organisation sectaire - atteint dans les années 1931-1932 une baisse considérable de son influence ${ }^{4}$ (Courtois et Lazar 1995 : 109, 119). Si l'on constate dans le manifeste des «restes» de cette période, notamment dans la phraséologie usitée, on remarque également qu'à aucun moment le PCF n'est mentionné, de même que le mot « communiste ». L'auteur du manifeste sait qu'il ne peut appeler au rassemblement des artistes et écrivains derrière la bannière d'un parti perçu comme ostracisant. Il proclame alors :

Qu'on n'attende pas de nous [...] on ne sait quel sectarisme qui nous ferait considérer les correspondants [correspondants ouvriers ou Rabcors] comme la source unique de toute littérature prolétarienne, qui jetterait l'interdit sur les écrivains et les artistes révolutionnaires venus d'autres horizons. (Anonyme [Paul Vaillant-Couturier] 1932)

6 Ce discours polémique se termine par l'annonce du programme de l'AEAR qui constitue plus de la moitié du manifeste et qui consiste en la propagation de la conception de l'art et de la littérature prolétarienne et révolutionnaire; à l'établissement, partout en France, de foyers d'écrivains et artistes révolutionnaires ; à la diffusion de cet art et de cette littérature par le biais d'«éditions, [de] livres, [de] brochures, [d']albums, [de] journaux, [de] bulletins, [de] feuilles volantes ».

7 Enfin, un appel à l'action est adressé aux lecteurs. Il s'achève par une incitation au groupement international de tous les travailleurs qui n'est pas sans rappeler l'injonction formulée par Marx et Engels dans le Manifeste du Parti communiste: «Prolétaires de tous les pays, unissez-vous » (Marx et Engels [1848] 1945 : 36). Au-delà du programme politique et idéologique qui est énoncé par l'AEAR, la structure même du texte est calquée sur celui de Marx et d'Engels. Seulement, si comme le dit Alain Meyer, le Manifeste du Parti communiste est devenu le «topos » du manifeste politique (Meyer 1980 : 38), il n'en reste pas moins que le manifeste de l'AEAR est avant tout un texte écrit pour une organisation d'écrivains et d'artistes.

8 Avec ce texte et la création de l'AEAR, le Parti communiste français s'implique durablement dans la vie artistique. Il s'agit alors d'étudier les modalités de cet engagement, les velléités du Parti en matière de création artistique et ses conséquences. Plus précisément, il sera question de savoir si l'association réussit à fédérer autour d'elle des artistes venus de divers horizons politiques et si elle y parvient par le biais d'un programme politique ou bien esthétique. 


\section{De l'exclusion au rassemblement}

9 Malgré l'esprit d'ouverture dont veut faire preuve le manifeste, celui-ci reste tout de même clivant. D'autres mouvements littéraires et artistiques pourtant favorables au communisme sont dénoncés, comme le groupe de la littérature prolétarienne et sa revue Nouvel Âge, dirigée par Henry Poulaille, qualifiés de «fascistes ${ }^{5}$ ». Pourtant, ce dernier et quelques autres membres de la littérature prolétarienne n'ont pas toujours été honnis par les communistes. L'Humanité a publié plusieurs récits de Poulaille, de Tristan Rémy et de Jean Tousseul (Lahanque 2002 : 174). Seulement, le refus de Poulaille de militer au sein du Parti et de suivre son orthodoxie fait de lui un " social-fasciste $^{6}$ " (175). Sa non-adhésion au Parti est perçue comme un renoncement à la révolution. Pour l'auteur du manifeste «la littérature prolétarienne sans solution révolutionnaire des antagonismes de classe ne peut être qu'un prêche d'obéissance et de soumission ».

L'hebdomadaire d'Henri Barbusse, Monde, est également dénoncé dans le manifeste pour sa "soumission à l'idéologie de la classe dominante " et sa «ligne contrerévolutionnaire ». Ces accusations contribuent à la mise à l'index de Monde, déjà annoncée en 1930 lors du congrès de Kharkov ${ }^{7}$. Son directeur, Barbusse, est membre du PCF depuis 1923, et en 1926 il est directeur littéraire de L'Humanité. L'année suivante, en novembre, lors de la Conférence des écrivains prolétariens et révolutionnaires qui se tient à Moscou, il est chargé de créer une organisation d'écrivains révolutionnaires en France dont l'organe serait Monde. Toutefois, il y renonce et va même jusqu'à revendiquer l'indépendance de sa revue vis-à-vis du PCF afin d'y attirer le plus grand nombre d'écrivains, artistes et intellectuels de gauche. L'hebdomadaire est alors qualifié de «Grand Bazar de l'idéologie » par Bruno Jasienski ${ }^{8}$ ou encore d'« ordure confusionnelle » par Louis Aragon".

11 En novembre 1930, le congrès de Kharkov condamne le groupe de la littérature prolétarienne, la revue Monde et son directeur, même si ce dernier reste défendu du bout des lèvres par les chefs de file du PCF qui voient en Barbusse le seul écrivain capable de fonder dans l'hexagone une association d'écrivains révolutionnaires ${ }^{10}$ (Bernard 1967). Seulement, les surréalistes, adhérents au Parti communiste depuis 1927, et qui participent par le biais d'Aragon et de Georges Sadoul au congrès de Kharkov, pensent que leur revient le droit de créer en France une organisation littéraire et artistique révolutionnaire ${ }^{11}$. Aragon écrit alors à André Breton depuis Kharkov :

Nous sommes d'accord avec les membres dirigeants [du congrès], qui disent à l'heure actuelle travailler avec des gens qui ont une plate-forme littéraire différente de la leur mais qui ont la même plate-forme politique qu'eux (c'est-à-dire en France les surréalistes) plutôt qu'avec des littérateurs qui semblent plus voisins d'eux littérairement mais qui politiquement sont des bourgeois (Barbusse et autres). [...] Nous comptons, Georges Sadoul et moi, sur votre confiance à tous, sur la tienne pour parler en votre nom à Kharkov où il nous sera peut-être donné de liquider, de contribuer à liquider Monde et tout ce qui s'ensuit. Ceci n'est pas chimérique. (Aragon cité par Juquin $2012: 511$ )

Mais au cours du congrès, Aragon retourne sa veste et proclame: « Nous ne sommes pas ici comme surréalistes, mais comme communistes ${ }^{12}$ " (Aragon cité par Juquin 2012 : 514). Toutefois, la franche opposition des surréalistes à Monde et à son directeur ne leur permet pas pour autant d'entrer dans les bonnes grâces du Parti qui se méfie d'eux ${ }^{13}$. C'est donc sans surprise que le congrès demande à Aragon et à Sadoul de se 
désolidariser des actions surréalistes, notamment du Second manifeste du surréalisme. Ils pourront ainsi suivre les « directives du Parti communiste, non seulement sur le plan politique, mais aussi sur le plan culturel ${ }^{14}$ ». L'AEAR reprend alors dans son manifeste la résolution de Kharkov qui :

[...] condamne à juste titre la base idéaliste du surréalisme [et] fait confiance à certains surréalistes pour abandonner leurs conceptions, et se rallier au matérialisme dialectique. Mais le surréalisme, en tant que méthode généralisable et que conception du monde, ne saurait être accepté par le prolétariat révolutionnaire ni accueilli dans nos rangs. (Anonyme [Paul Vaillant-Couturier] 1932)

L'AEAR appelle en effet les artistes à utiliser le matérialisme dialectique comme "méthode » de création, car «seul, le matérialisme dialectique met à découvert les rouages qui actionnent le monde. Seul, il donne à l'écrivain et à l'artiste l'explication de la réalité et les moyens de la transformer ». Les surréalistes, du moins Breton, y sont favorables. Dans le Second manifeste du surréalisme, en 1930, il donne son adhésion totale au principe du matérialisme historique. Cela ne l'empêche pas pour autant de vouloir " entreprendre le procès des notions de réalité et d'irréalité, de raison et de déraison, de réflexion et d'impulsion" (Breton 1929: 5). Cette volonté, même si Breton s'en défend, se rapproche considérablement d'une conception idéaliste (hégélienne) qui entre alors en contradiction avec le matérialisme du parti marxiste-léniniste. Si Karl Marx et Friedrich Engels adoptent le principe de la dialectique de George Hegel, ils délaissent cependant sa dimension idéaliste qui ne prend pas en compte «l'activité concrète humaine en tant que pratique » objective (Engels [1888] 1945 : 45). De cette façon, la méthode dialectique de Marx :

[...] diffère par la base de la méthode hégélienne, [...] elle en est même l'exact opposé. Pour Hegel, le mouvement de la pensée, qu'il personnifie sous le nom de l'Idée, est le démiurge de la réalité, laquelle n'est que la forme phénoménale de l'Idée. Pour moi [Karl Marx], au contraire, le mouvement de la pensée n'est que la réflexion du mouvement réel, transporté et transposé dans le cerveau de l'homme. (Marx [1867] $1938:$ 29)

Plus largement, l'idéalisme est rejeté par les communistes car il apparaît comme un frein à la révolution. La primauté qu'il accorde à l'idée plutôt qu'à l'activité (l'action) est perçue comme une invitation à se détourner de la lutte sociale et politique pour se retrancher dans une activité essentiellement phénoménale et théorique.

La grande place que consacre l'AEAR à la critique et à la dénonciation des autres groupes d'art et de littérature de gauche suppose qu'elle souhaite s'imposer dans la vie artistique par la force et de manière ostracisante. Son discours s'apparente alors à celui que tenait la RAPP (Association russe des écrivains prolétariens) quelques mois auparavant. Il est vrai que l'AEAR est créée sur le modèle de l'association russe qui depuis sa naissance en 1928 a pour but de favoriser l'éclosion d'une littérature "résolument industrialiste et strictement orthodoxe sur le plan idéologique " (Bernard 1967 : 521). En 1932, cette organisation est à bout de souffle, ses dérives sectaires ont pour conséquence d'affaiblir l'influence des écrivains soviétiques. Le Comité central du Parti communiste de l'Union soviétique décide alors de dissoudre la RAPP le 23 avril 1932, soit un mois après la création effective de l'AEAR. Un assouplissement dogmatique ressort de cette dissolution qui se traduit non plus par des déclarations d'intention (comme c'est le cas dans le manifeste) mais par une réelle ouverture des organisations artistiques et littéraires révolutionnaires aux compagnons de route. Toutefois, si des non-communistes peuvent intégrer l'AEAR, la mise en avant 
du matérialisme dialectique comme "méthode " à suivre pour les artistes et écrivains laisse apercevoir la volonté du Parti de s'immiscer concrètement dans la création artistique. L'étude de l'évolution de l'AEAR dans le temps permet d'en savoir plus sur ses volontés en matière d'art.

\section{Après le " manifeste " : la revue Commune (1933) et l'exposition des artistes révolutionnaires (1934)}

En juillet 1933, l'AEAR se dote d'un organe de diffusion, la revue Commune. Son premier comité directeur est composé de Barbusse, Vaillant-Couturier, Gide et Rolland. Le secrétariat de rédaction est formé quant à lui de Paul Nizan et d'Aragon. La présence de ces noms est significative de l'ouverture de l'association aux compagnons de route. Cet ensemble donne à la revue, et de ce fait à l'AEAR, l'image d'une organisation, certes ancrée à gauche, mais ouverte à différentes tendances. La revue souhaite alors :

[rendre] publique la lutte que mène l'AEAR, [et] fait connaître les éléments vivants d'une culture révolutionnaire qui se développe et dont les efforts et les résultats ne sauraient être isolés de l'action du prolétariat révolutionnaire ${ }^{15}$.

Dans son premier numéro, Commune annonce qu'elle envisage de toucher les «larges cercles intellectuels et petits-bourgeois que la crise a rendus perméables à la propagande révolutionnaire ${ }^{16} »$. Elle souhaite également recruter en masse des écrivains et des artistes qui sont jusqu'à ce jour encore réfractaires aux idées révolutionnaires. Cette action doit être menée «sur le plan idéologique et sur le plan des revendications professionnelles de ceux que nous désirons toucher " (ibid.). Elle vise donc à atteindre non seulement une catégorie sociale qui peut, par sa présence, gonfler les rangs des révolutionnaires, mais aussi une catégorie professionnelle qui, en rejoignant l'association, a la possibilité de devenir le promoteur de cette révolution. Ainsi, un an après un manifeste d'ordre plutôt théorique, Commune publie un plan stratégique de lutte qui annonce la mise en place du programme de l'AEAR. L'étude d'un événement organisé par l'association permet d'aborder plus concrètement la réalisation, ou non, de ce programme.

Du 27 janvier au 18 février 1934 se tient l'exposition des « Artistes révolutionnaires » à la Porte de Versailles. Une centaine de plasticiens y participent en exposant près de trois cents œuvres ${ }^{17}$ (Rosianu 1934). Parmi ces peintres, dessinateurs et sculpteurs, certains jouissent d'une reconnaissance nationale voire internationale comme Jean Carlu, Théodore Fried, Fernand Léger, André Lhote, Jacques Lipchitz, Jean Lurçat, Frans Masereel et Paul Signac. La jeune génération est également à l'honneur puisque sont présents Georges-Henri Adam, Maurice Estève, Édouard Pignon et Marcelo Pogolotti ${ }^{18}$.

Dans le manifeste de l'AEAR, il est énoncé que le rôle de l'association est de :

[...] propager autour de nous notre conception de la littérature et de l'art révolutionnaire prolétarien; souligner devant la classe ouvrière la nécessité et l'urgence de la création d'une littérature et d'un art prolétarien révolutionnaire en France. [Mais aussi de] demander aux artistes de mettre leur art au service direct des masses, de leurs démonstrations, de leurs luttes.

Le nombre important d'artistes et d'œuvres exposés dans un lieu populaire répond dans un premier temps à cette volonté de large diffusion. Ensuite, les titres de quelques-unes de ces œuvres comme Solidarité, Fraternisation ou encore La Prise du pouvoir, Le Prolétariat, Manifestation ${ }^{19}$, etc., renvoient à la deuxième volonté du manifeste 
de l'AEAR (qui est de mettre l'art «au service direct des masses, de leurs démonstrations, de leurs luttes»). De la même façon, la préface du catalogue de l'exposition écrite par Mathieu Rosianu confirme cette idée. Pour lui, l'art doit traduire "plastiquement le trouble social» mais aussi exalter la «foi du prolétariat en luimême" (Rosianu 1934 : n. p.). Ces consignes, assez larges, permettent à différentes tendances artistiques d'être représentées à cette exposition. On y trouve ainsi :

[des] peintres qui « parlent de leur malheur ", d'autres soucieux du contenu social et de lui seul, des œuvres apparemment sans volonté de signification sociale, que leurs auteurs bien intentionnés, nous obligent à croire sur parole, et aussi des peintres catégoriques, jeunes, optimistes (ibid., non paginé)

Il y a tout de même une cohésion dans cette diversité d'artistes et de tendances, qui repose sur un recul du " naturalisme-mot-à-mot » et de la mauvaise reproduction. L'art semble se tourner vers des "moyens d'expression plus évolués » car à une idéologie progressiste ne "sauraient correspondre des moyens d'expression périmés» (ibid.). Pourtant malgré cette diversité, la peinture figurative domine. Si certaines œuvres n'ont pas explicitement de "signification sociale ", comme la Joconde aux clés de Léger, elles représentent tout de même des éléments issus de la réalité visible.

\section{La question du réalisme}

L'appel au matérialisme dialectique lancé par l'AEAR en 1932 nous renseigne sur ses volontés esthétiques. Toutefois, jusqu'en 1934, il n'est pas question, dans Commune, de « méthode » de création tel que cela a pu être énoncé dans le manifeste de l'association. Pour cela il faut attendre le mois de juin et la préparation au Congrès des écrivains soviétiques (août 1934), qui se traduit dans Commune par la publication des « statuts de l'Union des écrivains soviétiques de l'URSS" et de l'article de Pavel Youdine et d'Alexandre Fadeev, «Le réalisme socialiste, méthode fondamentale de la littérature soviétique». Le réalisme socialiste est alors considéré comme l'expression de «l'assimilation critique de l'héritage du passé » et de l'« expérience accumulée par la construction victorieuse du socialisme et du relèvement culturel général » (Comité d'organisation de l'Union des écrivains soviétiques 1935 : 1150). Érigé comme méthode fondamentale de la littérature et de la critique soviétique, le réalisme socialiste exige de l'« artiste une peinture véridique et historiquement concrète de la réalité dans son développement révolutionnaire. Ce caractère de la description artistique doit s'allier au problème de l'éducation des masses laborieuses dans l'esprit du socialisme » (ibid.).

C'est peu ou prou ce que Youdine et Fadeev annoncent dans leur article, toutefois, ils préfèrent mettre l'accent sur la liberté qu'offre la nouvelle méthode de création, ce qui peut sembler paradoxal lorsque l'on incite les artistes à créer selon un procédé défini. Néanmoins, ils certifient que le réalisme socialiste n'est pas un « dogme, un recueil de lois limitant la création artistique réduisant toute la diversité des recherches et des formes à des commandements littéraires" (Youdine et Fadeev 1934: 1025). Cette liberté réside dans le fait que le réalisme socialiste est l'expression des nouvelles relations existantes entre l'artiste (en l'occurrence l'écrivain), une conception révolutionnaire du monde et une société socialiste en élaboration. Lors du congrès, l'idée que le réalisme socialiste est le résultat de l'interaction entre l'artiste et le développement révolutionnaire est également formulée par Nikolaï Boukharine et Andreï Jdanov. Ce dernier, qui est alors secrétaire général du PC(b)US, lui donne une 
définition qui marquera la création artistique soviétique et plus largement communiste pendant des décennies. Il souhaite que les artistes :

[connaissent] la vie socialiste afin de pouvoir la représenter véridiquement dans les œuvres d'art, la représenter non point de façon scolastique, morte, non pas simplement comme la «réalité » objective, mais représenter la réalité dans son développement révolutionnaire.

Et là, la vérité et le caractère historique concret de la représentation artistique doivent s'unir à la tâche de transformation idéologique et d'éducation des travailleurs dans l'esprit du socialisme. Cette méthode de la littérature et de la critique littéraire, c'est ce que nous appelons la méthode du réalisme socialiste. (Jdanov [1950] $1970: 5$ )

Quant à Boukharine, s'il revient également dans son intervention au Congrès sur le « sens social » du réalisme (Boukharine 1934 : 54-59), il insiste sur la base philosophique de cette nouvelle méthode de création qu'est le matérialisme dialectique. Seulement, si le matérialisme dialectique est pour lui une manière d'appréhender le monde à travers ses particularités scientifiques (sons, couleurs, électrons, ondes, etc.) et/ou sociales (économies, infrastructures, superstructures, classes, etc.), il considère que le réalisme socialiste doit s'attacher davantage au sensible, aux éléments intellectuels qui résultent d'une forme émotionnelle. Ce qui, encore une fois, garantit, en apparence, une certaine liberté à l'artiste.

L'ensemble de ces textes permet alors de situer le réalisme socialiste comme une émanation artistique du matérialisme dialectique. Ce dernier, on l'a vu, est le principe directeur de la pensée communiste. Plus que cela, il est considéré comme une " méthode généralisable » capable de donner «à l'écrivain et à l'artiste l'explication de la réalité et les moyens de la transformer " (Anonyme [Paul Vaillant-Couturier] 1932: 4). Le matérialisme dialectique, tel qu'il est défini par Marx et Engels est une conception du monde qui repose sur l'interaction entre la nature (tout ce qui est extérieur et indépendant de l'action humaine) et l'esprit humain (la pensée). De cette façon, pour Engels, le « monde ne doit pas être considéré comme un complexe de choses achevées, mais comme un complexe de processus " (Engels [1888] 1945 : 31) qui amène à un développement d'ordre progressif. Ainsi, l'artiste utilisant le matérialisme dialectique comme méthode de création a la possibilité, grâce au rapport qu'il entretient avec le monde, de le représenter et ainsi de participer à son processus de développement progressiste (la révolution). En même temps qu'il réalise cette opération, l'artiste se transforme aussi puisqu'il opère un changement au sein même du réel. Il est donc à la fois création et créateur en devenir. Ce qui fait dire à Marx que " pour l'homme socialiste, toute la prétendue histoire du monde n'est rien d'autre que la production de l'homme par le travail, donc le devenir de la nature de l'homme " (Freville et Thorez [1936] 1954 : 49).

Si le matérialisme dialectique constitue pendant près d'un an la base de la réflexion esthétique dans Commune, il reste néanmoins trop complexe et trop indéfini pour constituer à lui seul un principe de création. Dans le cadre de l'Exposition des artistes révolutionnaires de 1934, par exemple, il est peu probable que les œuvres soient réalisées selon le principe du matérialisme dialectique tel qu'il est énoncé par l'AEAR dans son manifeste. La définition du réalisme socialiste et son officialisation par les Soviétiques permet de répondre à un besoin patent. Les problèmes sont que, d'une part, cette définition est réalisée par des Soviétiques pour des Soviétiques et, d'autre part, que sa diffusion en France est effectuée par des hommes et des femmes de lettres 
puisque c'est une méthode créée avant tout pour la littérature. De cette façon, si dans le domaine des arts plastiques le terme "réalisme » est récurrent - notamment dans les réponses à l'enquête «Où va la peinture ${ }^{20}$ ? ", l'expression "réalisme socialiste » est absente des pages de Commune. Il faut attendre 1936 pour que le mot d'ordre de Jdanov soit prononcé dans le cadre d'un événement organisé par l'association qui succède à l'AEAR, la Maison de la culture ${ }^{21}$. Cette dernière organise en mai et juin une série de débats sur le réalisme en peinture. Le 16 mai, Aragon proclame :

[«les réalistes des jours du Front populaire »] sont des hommes d'un temps où les hommes ont entrepris de transformer la nature. C'est dire que la nature ne leur fournit que les éléments de leur art, mais qu'ils peignent pour que ces éléments deviennent profitables à l'homme, pour le devenir harmonieux de l'homme maître de la nature. [...] Ce réalisme cessera donc d'être un réalisme dominé par la nature, un naturalisme, pour être un réalisme, expression consciente des réalités sociales, et partie intégrante du combat qui modifiera ces réalités. En un mot, il sera un réalisme socialiste. (Aragon, dans Fauchereau [1936] 1987 : 96)

Ce n'est pas la première fois que l'ancien surréaliste s'engage à ce point sur la question du réalisme. En 1934, il publie Les Cloches de Bâle, qui est alors perçu par René Garmy (1934: 6) comme une tentative de littérature révolutionnaire. Un an plus tard, il fait paraître Pour un réalisme socialiste (1935) et s'impose en France comme le défenseur de cette théorie esthétique.

Les artistes qui participent aux débats de la Maison de la culture, manifestent, - quant à eux - des réticences, voire des résistances envers ce réalisme qu'ils considèrent comme inadaptable à la peinture française. Il apparaît que chaque artiste a sa propre conception du réalisme. Pour Lurçat, c'est une "disposition sentimentale », "une reprise de conscience de caractère avide et combatif d'un monde qui s'ouvre à l'espérance » (Lurçat, dans Fauchereau [1936] 1987 : 25). Pour Édouard Goerg, il existe plusieurs sortes de réalismes; selon lui, un artiste réaliste a une "lucidité non conformiste, de clairvoyance, d'audace, de prévision, d'anticipation " (Georg, dans Fauchereau [1936] 1987: 32). Le plus opposé aux thèses d'Aragon est sans conteste Léger. Ce dernier défend le "nouveau réalisme ", " fruit des progrès de la technique, du machinisme et des besoins nouveaux des classes jusqu'ici exclues du beau et de l'art » (Racine 2002 : 130). Le peintre pense, en effet, que si le « peuple » n'a pas été touché, au début $d u x^{e}$ siècle par les œuvres des "modernes ", ce n'est pas du fait du manque d'humanité des œuvres mais de "l'ordre social» qui ne permet pas au "peuple» d'accéder à "l'art», au "beau " (Léger, dans Fauchereau [1936] 1987 : 103). Afin de toucher les « masses », Léger propose un « nouveau réalisme » :

[...] qui a ses origines dans la vie moderne même, dans ses phénomènes constants, dans l'influence des objets fabriqués et géométriques, dans une transposition où l'imagination et le réel se croisent et s'enchevêtrent, mais où on a banni tout sentimentalisme littéraire et descriptif, tout dramatisme qui relève d'autres directions poétiques ou livresques. (Léger, dans Fauchereau [1936] 1987 : 105)

Surtout, il souhaite amener le peuple au «beau» en intégrant dans l'art (dans la peinture en particulier), des objets de la vie quotidienne. Avec la publicité et les arrangements en vitrine de magasin l'objet est en effet sublimé et revêt une dimension esthétique que souhaite capter Léger : « Les vitrines des magasins où l'objet isolé arrête et séduit l'acheteur: nouveau réalisme " (Léger, dans Fauchereau [1936] 1987 : 107). L'intégration de ces objets doit permettre au spectateur qui n'a pas eu accès à une culture artistique d'être touché par le «beau ». Cette beauté est alors celle du peuple et est éminemment réaliste. De plus, l'objet confère au réalisme une certaine forme 
d'objectivité que le retour au sujet prôné par Aragon ne permet pas. Léger développe ici ce qu'il avait déjà esquissé en 1935 dans sa réponse à l'enquête de Commune mais également dans d'autres écrits antérieurs ${ }^{22}$.

Les prises de position du peintre font réagir Aragon qui lui consacre une partie de son article « Le réalisme à l'ordre du jour ». Le " réalisme nouveau » de Léger est qualifié de « tarte à la crème » et d'art de soumission à la classe dominante. Pour Aragon, il n'est pas possible de répondre à une attente sociale en intégrant des objets de la vie quotidienne dans la peinture. De plus, cette intégration amène à la confusion puisque ces objets ne constituent pas le sujet de l'œuvre mais des éléments de composition. Enfin, cette sublimation de l'objet, industriel et/ou commercial, revient à sublimer l'ordre social contre lequel le peintre se bat, ce qui amène Aragon à écrire à l'attention de Léger: "Esclave, vous peignez vos chaînes» (Aragon 1936: 30). Le "nouveau réalisme » de Léger est alors en complète opposition avec celui que défend Aragon pour qui le réalisme socialiste doit s'envisager comme la représentation iconique du réel d'un point de vue de classe, c'est-à-dire que la réalité doit être appréhendée telle qu'elle est éprouvée par la classe ouvrière (dans ses souffrances et ses aspirations). autres artistes continuent à revendiquer un art contre le fascisme et pour la révolution. Il ressort de ces débats qu'il existe autant de réalismes que de peintres et malgré les volontés d'Aragon, le réalisme socialiste n'obtient pas le soutien espéré. Ces débats sont, finalement, révélateurs d'une chose : «l'esprit d'unanimisme du Front populaire » (Racine 2002 : 131). En effet, les artistes multiplient, au cours de ces débats, leur accord avec les mouvements sociaux en cours et les déclarations en faveur du Front populaire. Le temps où les artistes non adhérents au Parti étaient traités de "socio-fascistes » est révolu. L'époque est à l'ouverture politique, au front commun.

Depuis la création de l'AEAR en 1932 jusqu'à sa transformation en Maison de la culture en 1935 et la dissolution de cette dernière en 1939, ces organisations réussissent à fédérer autour d'elles non seulement des adhérents au Parti communiste, mais aussi des compagnons de route. Ce rassemblement, dont le point de départ est sans conteste le manifeste de 1932, doit permettre de poursuivre les buts du Parti sur le terrain de la culture. Il cherche en effet à étendre son contrôle dans le domaine des arts. Pour ce faire, il entend dans un premier temps déstabiliser les autres groupes de gauche, puis il évoque le matérialisme dialectique comme principe de création artistique. Seulement, le refus des artistes d'abandonner leur autonomie - comme on a pu le voir lors des débats de la Maison de la culture -, ainsi que la succession d'événements majeurs (émeutes fascistes, invasion de l'Éthiopie, guerre d'Espagne, etc.) font que le Parti n'attend plus des artistes qu'ils soient des « lutteurs du prolétariat » mais des remparts contre la guerre et le fascisme.

Il est alors possible de constater l'échec de la mise en pratique du "manifeste » notamment parce qu'il est basé sur un processus dialectique. De cette façon, les événements qui apparaissent après la publication du manifeste rendent caducs les volontés politiques de ce dernier et font de même pour leurs transpositions plastiques. Ce manifeste est donc, pour reprendre les mots de Louis Althusser :

[un texte qui avant tout] indique, à partir d'une multitude de données encore partielles, mais réelles une certaine direction utile et stimulante pour l'analyse et l'action, sans forcément rendre compte de l'ensemble des données et sans rendre compte de la réalité qu'il désigne. (Althusser, cité par Meyer 1980 :37) 


\section{BIBLIOGRAPHIE}

Anonyme, 1932, «L'Association des écrivains et artistes révolutionnaires tient son assemblée constitutive ", L'Humanité, 18 mars 1932, p. 2.

Anonyme [Vaillant-Couturier, Paul], 1932, « Le manifeste de l'Association des écrivains et artistes révolutionnaires ", L'Humanité, 22 et 29 mars 1932, p. 4.

Anonyme, 1933, « Document », Commune, $n^{\circ}$ 1, juillet 1933, p. 87-95.

Ambroise, Jean-Charles, 1998, Henry Poulaille et le mouvement français pour la littérature prolétarienne. Position littéraire, représentations, prises de positions politiques : 1925-1944, thèse d'État, Université Rennes I.

Aragon, Louis et Sadoul, Georges, 1930, lettre « autocritique » adressée au secrétariat de l'Union internationale des écrivains révolutionnaires, Moscou, $1^{\mathrm{er}}$ décembre 1930, [En ligne], http:// melusine-surrealisme.fr/site/Tracts_surr_2009/Tracts_I_2009.htm, consulté le 15 septembre 2018.

Aragon, Louis, 1935, Pour un réalisme socialiste, Paris, Denoël et Steele.

Aragon, Louis, 1936, « Le réalisme à l'ordre du jour », Commune, n 37, septembre 1936, p. 30.

Bernard, Jean-Pierre, 1967, « Le Parti communiste français et les problèmes littéraires (1920-1939) », Revue française de science politique, $17^{\circledR}$ année, $n^{\circ}$ 3, p. 520-544.

Boukharine, Nikolaï, 1934, «Le réalisme socialiste », Commune, n 13-14, septembre-octobre 1934, p. 54-59.

Breton, André, 1929, « Second Manifeste du Surréalisme », La Révolution Surréaliste, $\mathrm{n}^{\circ} 12$, décembre 1929, p. 1-17.

Comité d'organisation de l'Union des écrivains soviétiques de l'URSS, 1934, «Les statuts des écrivains soviétiques de l'URSS », Commune, $\mathrm{n}^{\circ}$ 10, juin 1934, p. 1149-1152.

Commune, «Commune. Revue de l'Association des écrivains et artistes Révolutionnaires », Commune, $\mathrm{n}^{\circ}$ 1, juillet 1933, n.p.

Courtois, Stéphane et Lazar, Marc, 1995, Histoire du Parti communiste français, Paris, PUF.

Engels, Friedrich, [1888] 1945, Ludwig Feuerbach et la fin de la philosophie classique allemande, Paris, Éditions sociales, coll. « Les éléments du communisme».

Fauchereau, Serge (préf.), Léger, Fernand et al., [1936] 1987, La Querelle du réalisme, Léger, Le Corbusier, Lurçat, Aragon, Lhote, Goerg, Cassou, Delaunay, Paris, Éditions Cercles d'art, coll. « Diagonales ».

Garmy, René, 1934, « Les Cloches de Bâle », L’Humanité, 31 décembre 1934, p. 6.

Jdanov, Andreï, [1950] 1970, « Discours au Premier Congrès des écrivains soviétiques, 17 août 1934 », dans Sur la littérature, la philosophie et la musique, Paris, Éditions Norman Béthune.

Juquin, Pierre, 2012, Aragon : un destin français, t. I, « Le temps des rêves, 1897-1939 », Paris, La Martinière.

Klein, Wolfgang, 1988, Commune : revue pour la défense de la culture. 1933-1939, Paris, Éditions du CNRS, coll. « Esthétique et politique». 
Laglenne, Jean-François et al., 1935, « Où va la peinture ? ", Commune, n 22, juin 1935, p. 1118-1134.

Lahanque, Reynald, 2002, Le Réalisme socialiste en France (1934-1954), thèse d'État, Université Nancy II.

Léger, Fernand et al., 1935, « Où va la peinture ?», Commune, nº 21, mai 1935, p. 937-960.

Léger, Fernand, [1965] 2004, Fonctions de la peinture, Paris, Gallimard.

Marx, Karl, [1867] 1938, Le Capital, t. I, « Le développement de la production capitaliste », Paris, Bureau d'Éditions.

Marx, Karl et Engels, Friedrich, [1848] 1945, Manifeste du Parti communiste, Paris, Éditions sociales, coll. « Les éléments du communisme».

Marx, Karl et Engels, Friedrich, avec une introduction de Jean Fréville et Maurice Thorez, [1936] 1954, Sur la littérature et l'art, Paris, Éditions Sociales.

Meyer, Alain, 1980, «Le manifeste politique : modèle pur ou pratique impure ? ", Littérature, $\mathrm{n}^{\circ}$ 39, p. 29-38.

Morel, Jean-Pierre, 1985, Le Roman insupportable. L'Internationale littéraire et la France, 1920-1932, Paris, Gallimard, coll. « Bibliothèque des idées ».

Racine, Nicole, 2002, « “La Querelle du Réalisme” (1935-1936) », Sociétés et Représentations, n 15, décembre 2002, p. 113-131.

Rosianu, Mathieu, 1934, (pref.), Exposition des Artistes Révolutionnaires, catalogue de l'exposition, Paris, Porte de Versailles, 27 janvier-18 février 1934, Paris, Association des écrivains et artistes révolutionnaires.

Youdine, Paul et Fadeev, Alexandre, 1934, Commune, nº 10, juin 1934, p. 1025-1030.

\section{NOTES}

1. Voir également, Anonyme, «L'Association des écrivains et artistes révolutionnaires tient son assemblée constitutive ", L'Humanité, 18 mars 1932, p. 2.

2. Voir à ce propos Morel (1985).

3. Lorsque la référence n'est pas précisée, elle renvoie au «Manifeste de l'Association des écrivains et artistes révolutionnaires ".

4. Aux élections législatives de 1928 le PCF perd douze sièges par rapport à 1924. Cette désaffection est confirmée par les élections de mai 1932 lors desquelles le Parti n'obtient que $8,4 \%$ des suffrages exprimés et ne fait élire que dix députés. Cette baisse de l'influence communiste se constate également par la chute du nombre de ses adhérents: plus de $45 \%$ de perte entre 1927 et 1931.

5. En ce qui concerne les relations entre Henry Poulaille, la littérature prolétarienne et le PCF voir Ambroise (1998).

6. Il écrit en 1931 que si « la littérature prolétarienne [peut] être marxiste », il ne pense pas, en revanche, que «seule la littérature marxiste soit prolétarienne » (Poulaille, cité par Lahanque 2002 : 175).

7. Le congrès, organisé par l'Internationale communiste, en novembre 1930, conduit à la fondation de l'« Union internationale des écrivains révolutionnaires » (UIER) dont émane l'AEAR. 8. « Au Grand Bazar de l'idéologie », Vestnik, nº 5, septembre-octobre 1929. À ce sujet voir Morel (1985 : 281-288). 
9. La Révolution surréaliste, $\mathrm{n}^{\circ} 12$, décembre 1929, p. 33.

10. Comme le fait remarquer Jean-Pierre Bernard, Henri Barbusse est certes attaqué lors de ce congrès en tant que symbole de l'écrivain communiste mais son aura internationale lui vaut d'être épargné en tant qu'homme.

11. Déjà, au cours de l'année 1930, André Breton et André Thirion tentent d'organiser l'Association des artistes et des écrivains révolutionnaires (AAER). André Thirion envoie à André Breton les statuts de l'association et son texte manifeste début décembre 1930, mais il semble que ce projet soit plus ancien, de quelques mois. Une chose est sûre c'est que le projet de l'AAER est antérieur au congrès de Kharkov qui s'ouvre le 6 novembre et qui définit la mise en place de l'AEAR. À ce propos voir Juquin (2012). Voir également le site http://www.andrebreton.fr où sont reproduits dans le dossier «AAER/AEAR» les documents concernant le projet de cette association.

12. Cette phrase sera par la suite connue des surréalistes restés à Paris et participera au divorce entre Louis Aragon et André Breton.

13. En 1929, le Bureau international de la littérature révolutionnaire attaquait les surréalistes en ces termes : « Malgré leurs renvois grandiloquents au marxisme et à la pensée dialectique, leurs écrits se distinguent, en plus de leur phraséologie d'extrême gauche, par un éclectisme absolu et une imagination romantique de petit-bourgeois " (Anonyme, Vestnik, mai-juin 1929, cité par Juquin 2012 : 517). La méfiance des communistes à l'égard des surréalistes se retrouve également en France. Le 15 novembre 1930, L'Humanité attaque les surréalistes en ces termes : «Une revue qui s'intitule Le Surréalisme au service de la révolution vient de publier un article intitulé "De Barbusse à Coty" qui est une injure à l'adresse de notre camarade. L'activité surréaliste, qui ne nous a jamais intéressés, n'a rien en commun ni avec les buts, ni avec les méthodes du prolétariat » (Anonyme, « Deux mises au point », L’Humanité, 15 novembre 1930, p. 2).

14. Aragon et Sadoul (1930), http://melusine-surrealisme.fr/site/Tracts_surr_2009/ Tracts_I_2009.htm.

15. «Commune. Revue de l'Association des écrivains et artistes révolutionnaires », Commune, $\mathrm{n}^{\circ} 1$, juillet 1933, n. p.

16. Anonyme, « Document ", Commune, $\mathrm{n}^{\circ} 1, \mathrm{p} .87$.

17. Notons également la présence à cette exposition de différents groupes comme le « groupe des Indélicats» et «la Jeune Révolution ». Plusieurs artistes hollandais présentent également des travaux. Enfin, des affiches d'URSS, des journaux d'usines, de photos, etc. sont exposés. Voir Rosianu (1934).

18. Ces artistes exposent respectivement: Art graphique (s.n.); Enterrement, Tuberculeux, Vielle femme, Le lit, Joueurs aux quilles, Le pain, Les cosaques de Paris, Toupie, Sans travail, Mutilé de guerre; La Joconde aux clés et quatre gouaches (s.n.) ; 14 juillet ; David et Goliath ; L'émeute ; Le matelot, Le mort (aquarelle); Pantin, Saint-Denis, Bercy (aquarelles) ; Histoire de Donia Rosenzweig, Visite du ministre Paul Reynaud en Indochine, six dessins (s.n.); Guerre, deux peintures (s.n.) ; Meeting et Oudarnik; Paysage cubain, Cadeau à la maîtresse, six dessins.

19. Ces œuvres sont, respectivement, d'Antek, de Robert Augros, de Mathieu Rosianu, de Deupe et d'Henri.

20. «Où va la peinture ? ", Commune, $\mathrm{n}^{\circ}$ 21, mai 1935, p. 937-960 et $\mathrm{n}^{\circ}$ 22, juin 1935, p. 1118-1134. Conversations recueillies par Jean Cassou, René Crevel, Aragon, Champfleury et Proudhon. Opinions d'Honoré Daumier, Gustave Courbet, Horace Vernet, Jacques-Émile Blache, André Derain, Paul Signac, Raoul Dufy, André Lhote, Marie Laurencin, Fernand Léger, Frans Masereel, Robert Delaunay, Jean Lurçat, Jean-François Laglenne, Christian Bérard, Yves Tanguy, Valentine Hugo, Max Ernst, Amédée Ozenfant, Jean-Louis Garcin, André Marchand et Jean Carlu.

21. Créée en 1935, l'Association des Maisons de la culture fédère différentes associations artistiques et culturelles proches du Front populaire dont l'Association des Peintres, Sculpteurs et Graveurs de la Maison de la culture (qui émane des Artistes révolutionnaires de l'AEAR). Ce 
mouvement est censé représenter sur le front de la culture la politique de la main tendue voulue par le Parti communiste à l'heure de la création du Front populaire.

22. Notamment dans «La Roue, sa valeur plastique» (1922), «Le ballet-spectacle, l'objetspectacle " (1923), «À propos de l'élément mécanique » (1923), "L'esthétique de la machine, l'ordre géométrique et le vrai» (1924), «La rue, objets, spectacles» (1928), «Un nouveau réalisme, la couleur pure et l'objet » (1935). Ces textes sont reproduits dans Fernand Léger ([1965] 2004).

\section{RÉSUMÉS}

Depuis sa création en 1932 en passant par son intégration au mouvement des Maisons de la culture en 1935 et jusqu'à la dissolution de celle-ci en 1939, l'Association des écrivains et artistes révolutionnaires (AEAR) s'impose comme une plateforme littéraire et artistique de premier ordre en France. Fondée par des intellectuels communistes et des compagnons de route selon des directives soviétiques, l'AEAR veut être une organisation ouverte à l'ensemble des forces de gauche dans le but de lutter, sur le front de la culture, contre la guerre et le fascisme. Le manifeste de l'AEAR, publié en mars 1932 dans L'Humanité indique la stratégie à adopter pour mener à bien cette lutte. Ce manifeste est une base sur laquelle s'ajoutent, avec le temps, des événements politiques et culturels qui viennent altérer sa stratégie originelle. L'étude de cette stratégie, de son évolution et de ses modalités d'application est l'objet de ce travail.

Since its creation in 1932 through its integration to the mouvement des Maisons de la culture (Houses of culture's movement) in 1935 and until the dissolution of the latter in 1939, the Association des Écrivains et Artistes révolutionnaires (AEAR) [Revolutionary Writers and Artists' Association] stands out as one of the most significant literary and art platforms in France. Founded by communist intellectuals and fellow travellers according to Soviet directives, the AEAR had the vocation of being an organization open to all left-wing forces in order to fight against war and fascism on the cultural front. The manifesto of the AEAR, published in March 1932 in the newspaper L'Humanité, indicates the strategy to carry out this fight. This manifesto is a foundation on which were added, over the years, political and cultural events, which altered its original strategy. The study of this strategy, its evolution, and the implementing of its rules constitute the topic of this work.

\section{INDEX}

Mots-clés : art, manifeste, communisme, révolution, réalisme

Keywords : art, manifesto, communism, revolution, realism

\section{AUTEUR}

\section{GWENN RIOU}

Aix-Marseille Université 